\title{
THE ANALYZES OF PDOP FACTORS FOR A ZIGBEE GROUND - BASED AUGMENTATION SYSTEMS
}

\author{
Artur Janowski ${ }^{1}$ \\ Jacek Rapinski ${ }^{2}$ \\ ${ }^{1}$ Institute of Geodesy, Faculty of Geodesy, Geospatial and Civil Engineering University of Warmia and Mazury in Olsztyn Poland \\ ${ }^{2}$ Institute of Geodesy, Faculty of Geodesy, Geospatial and Civil Engineering University of Warmia and Mazury in Olsztyn Poland \\ These authors contributed equally to this work.
}

\begin{abstract}
This paper presents the analysis of PDOP factors for a ZigBee ground - based augmentation systems. It presents the idea of such a system followed by the results of assessment of application in Gdansk Marina. The results of the experiment show that the application of ZigBee can significantly improve PDOP value in harsh measurement environment. The analysis shows that it is possible to select an optimal location of ground-based transceiver on the basis of predicted trajectory and obstructions measured with laser scanning.
\end{abstract}

Keywords: point cloud processing, GNSS, ZigBee, GBAS, PDOP, navigation in harbor

\section{INTRODUCTION}

The need for localization based services in almost every place is constantly growing. GNSS systems are widely used to provide position, velocity and time solutions. The major drawback of these systems is the lack of satellite signals when the sky is obstructed $[14,20,26]$. For this reason, ground based augmentation systems (GBAS) [5] are gaining more and more attention in the modern world. GBAS systems are meant to improve the GNSS system performance in the terms of availability, accuracy and continuity. Dedicated GBAS systems are widely used to support precision aircraft approach operations. GBAS can be created in various ways from differential corrections transmitted using very high frequency data broadcast to pseudolite systems.

The most crucial part of ship navigation is in the harbor or in a water channel. Limited space and high obstacles on the shore are making the GNSS navigation less reliable then in the open sea (where the requirements for the navigation are smaller). In order to mitigate the limitations of GNSS in such places a GBAS system can be introduced.
Harbor infrastructure is an important part of the economy. A seaport could operate freely, if the following criteria are fulfilled:

- access to other kinds of transport,

- winds and currents protection,

- sufficiently deep water channel,

- storage space of goods (warehouses, storage yards, tanks, siloes, with different strength, internal structure, height and capacity with a huge influence to the use of harbor space) $[1,2,4,7,9,7,14,16-17]$

In this paper we would like to present the impact of a lowcost, ZigBee based GBAS on the accuracy and availability of GNSS in a harbor. ZigBee is one of the IEEE 802.15.5 low-rate wireless personal area networks (LR-WPAN) [13, 23]. It is a small, low-power digital radio used mainly for industrial sensor applications. It is designed for embedded application with low power consumption (it can operate up to few years on a single AA battery) and low data rates. Despite the main purpose of ZigBee devices (which is wireless communication), some devices are equipped with ranging capabilities. Successful tests of such a device are presented in $[15,18,19]$ AT233RF device presented in those papers 
can measure a distance with decimeter accuracy in the $\sim 300$ meters range. The application of such a device next to the ships GNSS antenna can significantly improve the availability and accuracy of positioning in the area covered with the ZigBee signals. The idea of the ZigBee GBAS system is presented in Figure 1.

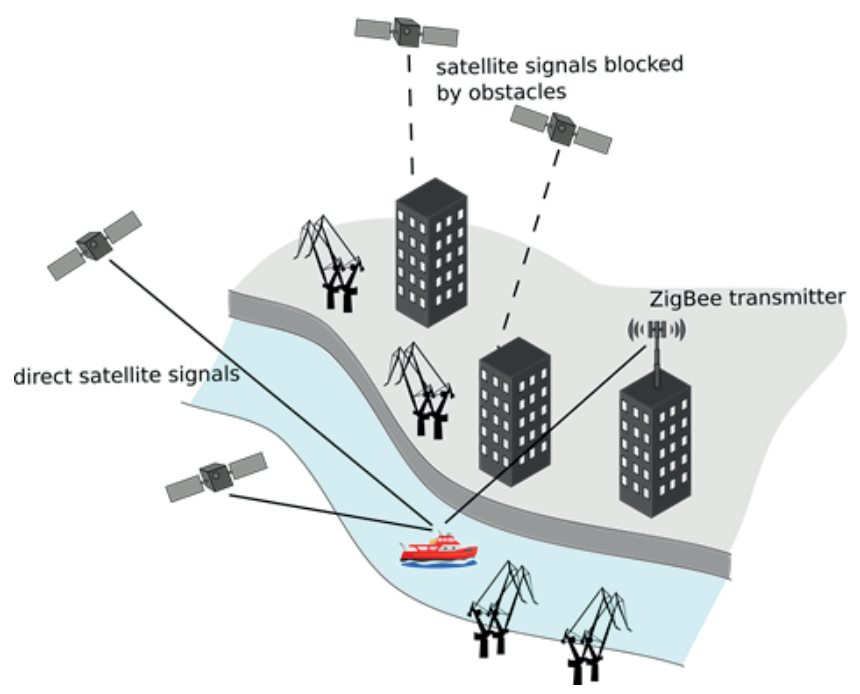

Fig 1. The idea of ZigBee GBAS

To evaluate the impact of transmitters geometry on the final position error DOP (Dilution Of Precision) factors are commonly used $[10,25]$. Smaller DOP value indicates better geometry of satellites [3]. Minimum value of PDOP is about 1.63 for four satellites. If design matrix of a GNSS point positioning solution is defined as:

$$
P D O P=\sqrt{\sigma_{x}^{2}+\sigma_{y}^{2}+\sigma_{z}^{2}}
$$

where

$$
\sigma_{x}^{2}=Q_{11}, \sigma_{y}^{2}=Q_{22}, \sigma_{z}^{2}=Q_{33}
$$

and

$$
Q=\left(A^{T} A\right)^{-1}
$$

Matrix A is defined by:

$$
A=\left[\begin{array}{cccc}
a_{11} & a_{12} & a_{13} & 1 \\
a_{21} & a_{22} & a_{23} & 1 \\
a_{31} & a_{32} & a_{33} & 1 \\
\vdots & & & \\
a_{n 1} & a_{n 2} & a_{n 3} & 1
\end{array}\right]
$$

where

$$
a_{i 1}=\frac{x_{i}-x_{u}}{\varrho_{i}}, a_{i 2}=\frac{y_{i}-y_{u}}{\varrho_{i}}, a_{i 3}=\frac{z_{i}-z_{u}}{\varrho_{i}}
$$

and

$$
\varrho_{i}=\sqrt{\left(x_{i}-x_{u}\right)^{2}+\left(y_{i}-y_{u}\right)^{2}+\left(z_{i}-z_{u}\right)^{2}}
$$

Where:

$x_{i}, y_{i}, z_{i}$ - coordinates of $\mathrm{i}$-th satellite,

$x_{u}, y_{u}, z_{u}$ - coordinates of receiver

Since ZigBee transmitter does not depend on the GPS time, an additional ground based transmitter will not improve TDOP parameter. Therefore, we have focused on the PDOP only.

A major factor influencing satellites visibility (and PDOP factors) are obstructions of the sky. Knowing the satellites almanac and obstructions at a certain place, we can determine the DOP factors and investigate if an additional ground based transmitter would improve positioning accuracy. At the same time, the decision about the optimal (from the PDOP point of view) localization of the transmitter can be made.

In order to verify if the optimal position of a GBAS transmitter can be selected on the basis of the previous assumptions, an experiment was conducted.

\section{EXPERIMENT}

The experiment took place in Gdansk (Poland) in the Gdansk Marina next to the Szafarnia street (Fig.2). The difficulty that had to be overcome, was to determine the objects obstructing GPS signals at certain position and time. The possibility of using the conventional solutions based on $3 \mathrm{D}$ or $2.5 \mathrm{D}$ maps were rejected because of the generalization of the description of the height coordinate especially in the case of buildings.

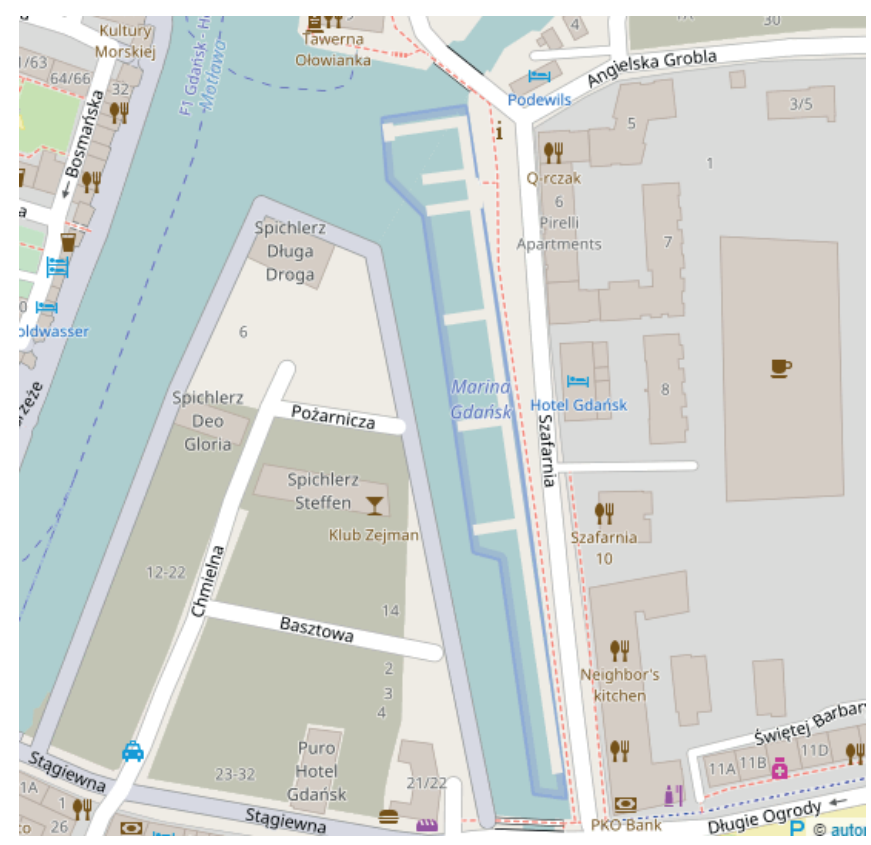

Fig. 2 The Marina Gdansk

One possible solution is the use of a DEM (digital elevation model), but even here the process of generalization can produce some significant errors from the satellite - receiver line of sight determination point of view. Therefore, the laser 
scanner measurement results were used. The measurements were performed using Riegl WZ 400 TLS (Terrestrial Laser Scanner). The measurement data was obtained in horizontal coordinate systems PL-2000 zone 6 (EPSG:2177) and vertical PL-KRON86-NH. The resulting point cloud is depicted in Figure 3.

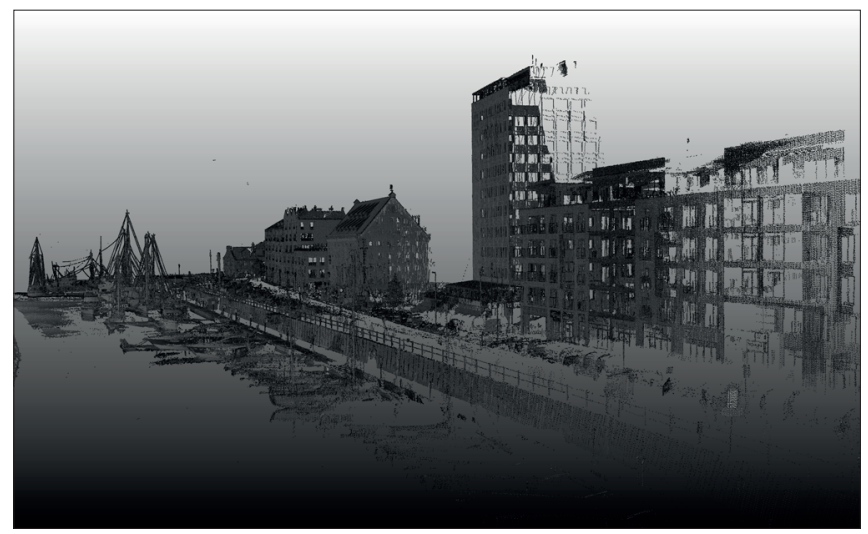

Fig. 3 The point cloud view

The study area had about $130 \times 370 \mathrm{~m}$ of a rectangular shape. At the beginning of the work all points of elements that did not have a permanent character in the measured space (floating or moored vessels, shapes of people, etc.) have been removed. In this case this task was completed manually, but in more complex situations one can apply one of the automatic methods of point clouds reduction [11]. The final result was a cloud of points containing more than $4,000,000$ points. The assumed test trajectory is shown in Figure 4. This trajectory consists of 13 points (numbered from one to thirteen from north to south). For each of these points the line of sight to every GPS satellite was tested. Figure 4 shows the test area overlapped with point cloud, trajectory and possible ZigBee locations. The numbers next to the trajectory points refers to the number of visible satellites at this point. Two points marked in red (point 12 and 13) are the points with the worst constellation of satellites.

The analysis of the visibility of the receiver-satellite including obstructions described by the cloud of points requires all spatial data to be in the same coordinate system. WGS84 ECEF (Earth Centered Earth Fixed) system (EPSG code: 4328) was selected. With the position of the GNSS receiver, satellites and point cloud in this coordinate system, the satellite visibility was validated. In the validation process only two possible situations were determined - satellite is visibility or not. No gradation of the quality of the visibility was introduced. In this paper, it is assumed that if there is more than 300 TLS points in the cylinder of radius $0.5 \mathrm{~m}$ expanded along the receiver - satellite line of sight (LOS) section, the satellite is obstructed. With this basic assumption, for each visibility test it is required to analyze the whole point cloud ( for each LOS). With millions of points this would be a very time-consuming task. Therefore the data must be prepared for such analysis by spatial indexation. For this purpose Database Management System (DBMS) PostgreSQL with PostGIS extension was adapted [28].

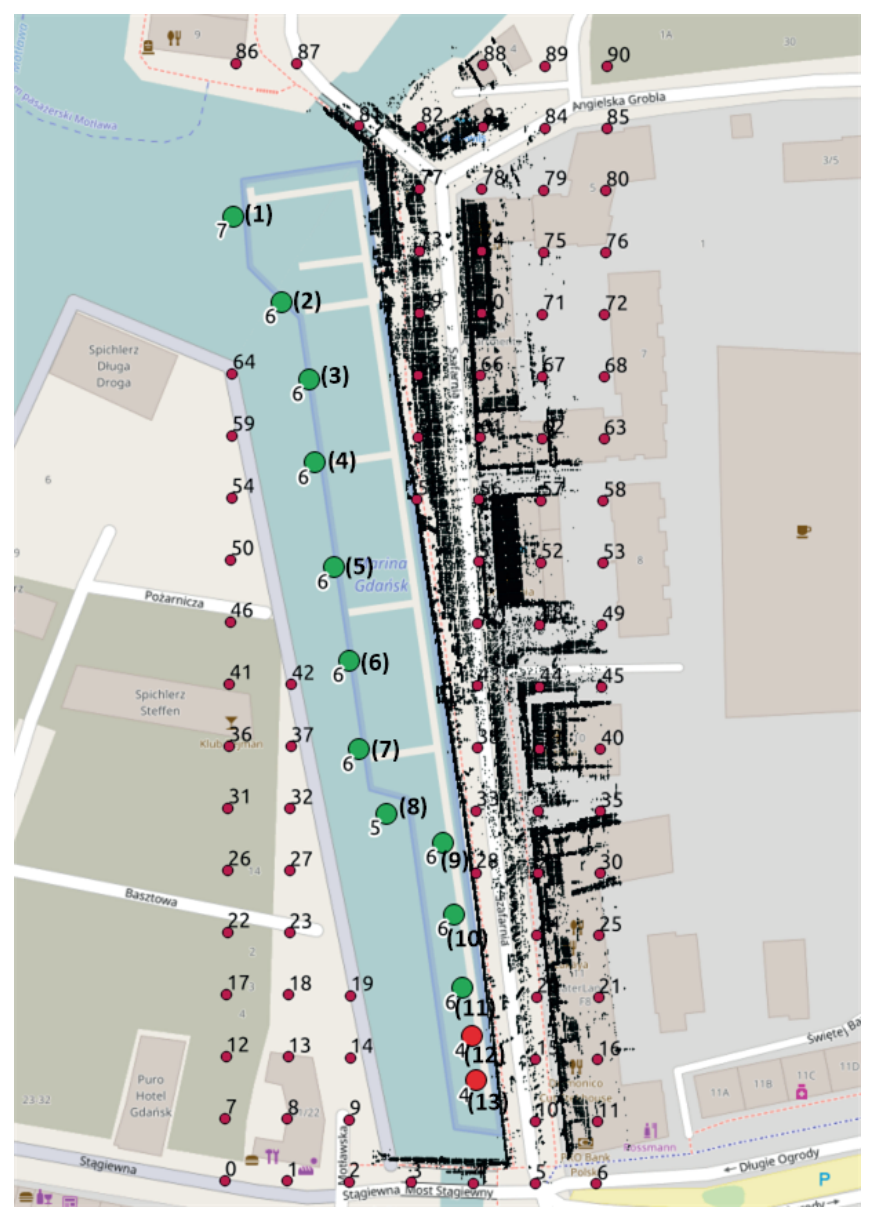

Fig. 4 The test area overlapped with point cloud, trajectory and possible locations of a ZigBee transceiver

The use of PostGIS gives the possibility to perform advanced and optimized spatial analysis and transformations of stored objects between coordinate systems and finding the relations between spatial objects described in a variety of coordinate systems. The use of PostGIS to find a number of points contained in the cylinder mentioned above is trivial. It is limited to application of st_3ddistnace function which returns the 3-dimensional Cartesian minimum distance between two geometries. A 3D section connecting GPS receiver with consecutive satellites and a set of LiDAR points were used as an input for this function. As a result, all of the distances from LiDAR points to this section were calculated. The selection of obstructions were made on the following assumption: if there is less than 5 points with distance smaller than $2.5 \mathrm{~m}$ the line of sight exists. Otherwise the satellite is obstructed. Tests were performed for a GPS constellation from 2016-05-20 starting from 18:00 UTC depicted in Figure 5. 


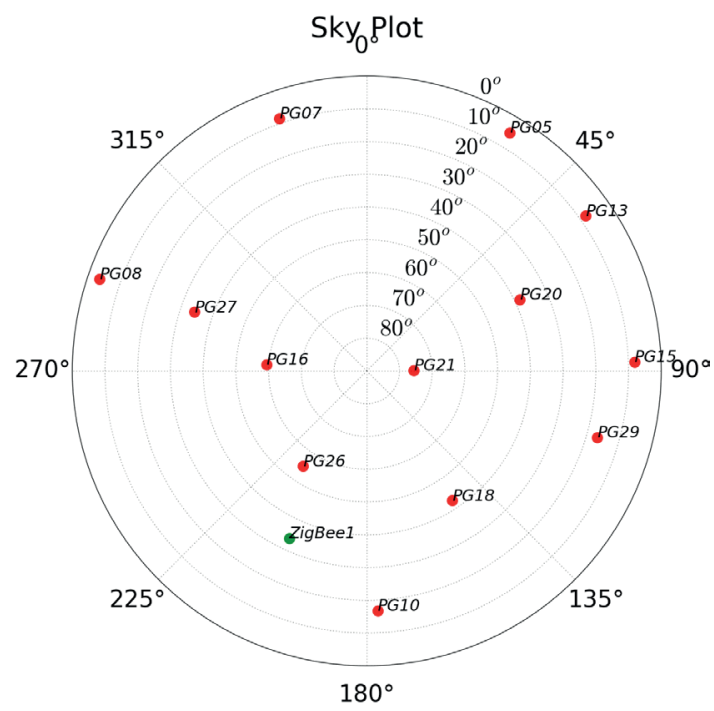

Fig. 5 The GPS constellation during analyzed test (The elevation and azimuth values are given in degrees unit. $\pi \mathrm{rad}=180^{\circ}$ )

\section{RESULTS}

The number of satellites and resulting PDOP values for each trajectory point is depicted in Figure 6.

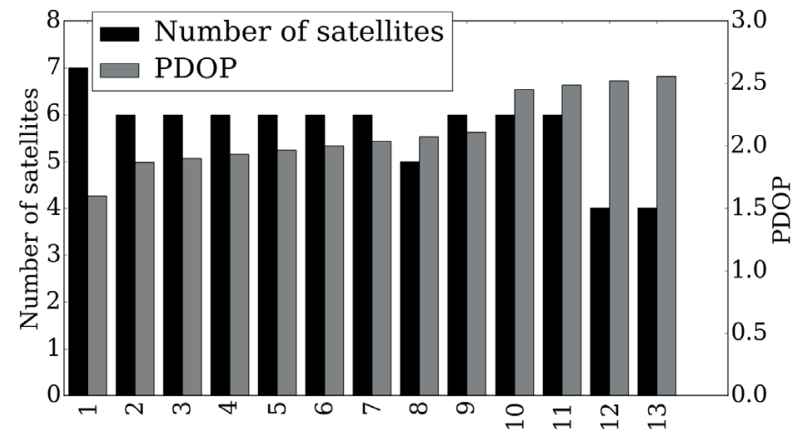

Fig. 6 The resulting PDOP values for each trajectory point

The location of the ZigBee transmitter impacts the PDOP value. In order to select the position for which the PDOP is minimal, a grid of possible Zigbee locations was stretched on the test area. Spacing of grid points was set to $20 \mathrm{~m}$. The height at which ZigBee transceivers were located was set to $18 \mathrm{~m}$ above the sea level. For each possible Zigbee location thirteen PDOP values were calculated assuming receiver position in consecutive points of the trajectory. As a result, thirteen heat maps (created for a possible locations of a single transceiver) were created. In Figure 7 dots represent the possible Zigbee locations, while underlying color depicts the PDOP values. PDOP values were calculate for each possible ZigBee location (taking obstructions in to account) and heat maps were interpolated (multiquadric radial basis function) [6]

In addition, the cumulative PDOP were calculated and depicted. We have defined a cumulative PDOP value as:

$$
c P D O P=\frac{\sum_{i=1}^{n} P D O P_{i}}{n}
$$

Where PDOP $_{i}$ is PDOP for i-th trajectory position and $\mathrm{n}$ is the number of points in the trajectory. This parameter depicts the average value of PDOP for the entire trajectory. The resulting heat maps show small deviations of the PDOP value $(1.2-2.4)$. This is caused by many satellites still visible on the eastern part of the sky.
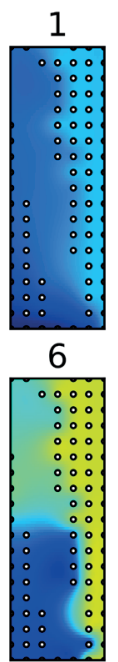

11
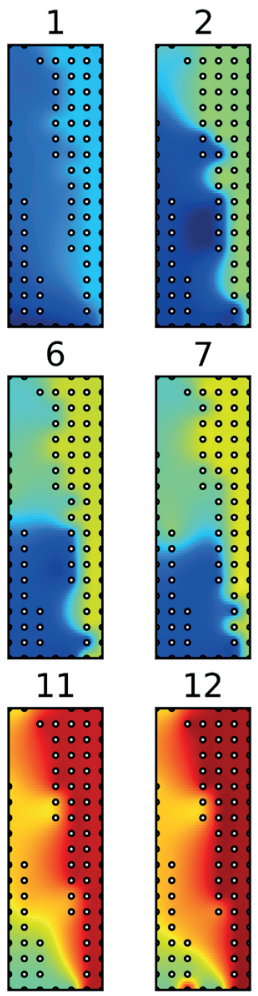

12
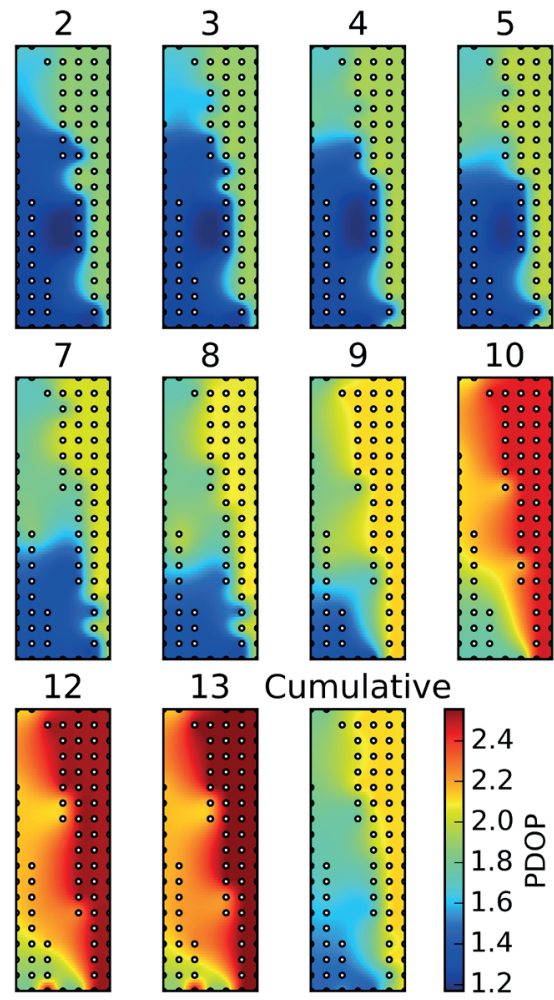

Fig. 7 The heat map for PDOP values

To simulate more difficult conditions, free satellites (namely PG08, PG27 and PG16) where removed from the constellation. Resulting PDOPs and numbers of satellite in view without additional ZigBee are depicted in Figure 8.

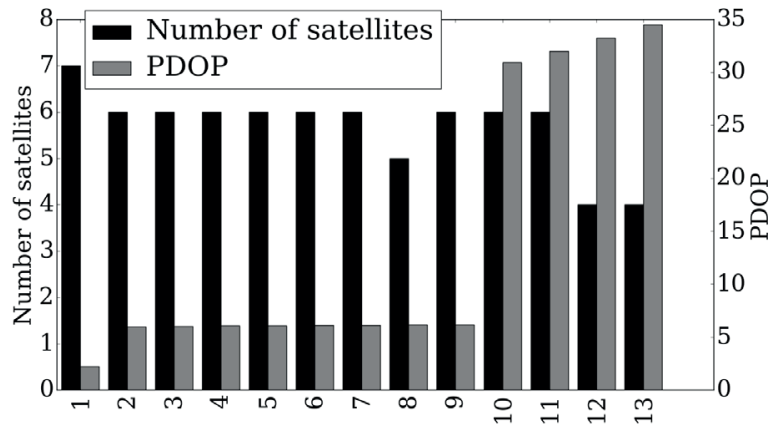

Fig. 8 The resulting PDOP values for each trajectory point (second scenario) 
It is clear that when the trajectory is getting closer to wharf, the number of satellites decreases and PDOP value increases. Without ZigBee the PDOP value at the end of the trajectory reaches 35 - Figure 8. Results of calculated PDOP values for reduced number of satellites and simulated ZigBee locations are depicted in Figure 9.

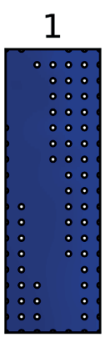

6

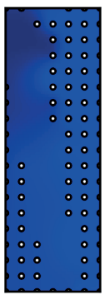

11
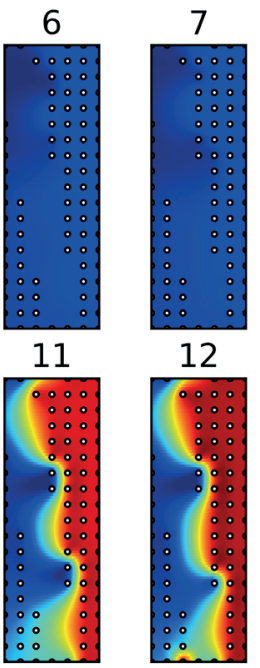

12
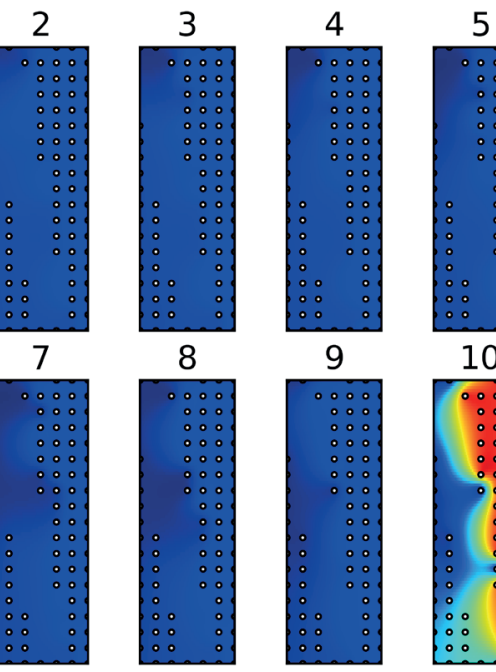

9
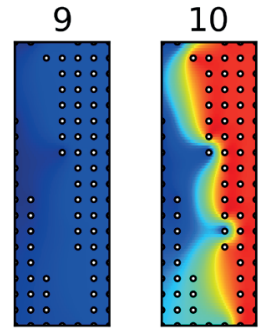

13 Cumulative
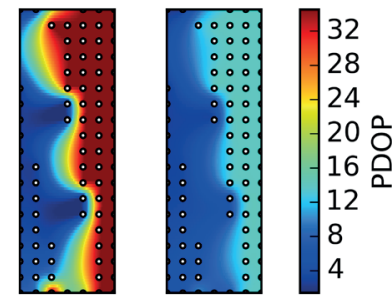

Fig. 9 The heat map for PDOP values (second scenario)

\section{DISCUSSION}

Analyzing Figure 9 one can notice possible transmitter location for which PDOP value is low for each point of trajectory (point on the blue background). The common point with low PDOP for each trajectory point is point number 55. The differences between PDOP values (at point 55) for the case with and without ZigBee for each point of the trajectory are depicted in Figure 10.

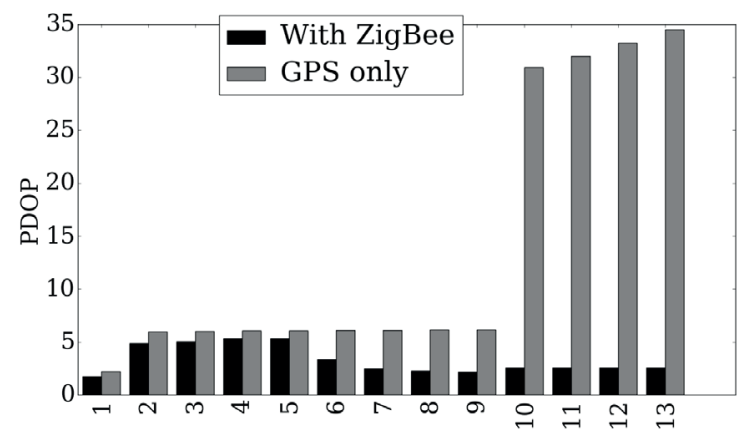

Fig. 10 The resulting PDOP values for each trajectory point (scenarios comparison)
Improvement of PDOP value is much bigger for the points at the end of the trajectory where the number of satellites in view decreases significantly.

\section{CONCLUSIONS}

Application of ZigBee based GBAS can improve the geometry of a positioning solution. The improvement is significant in the most unfavorable environment. When the number of satellites in view is satisfactory and their geometry is good, the application of GBAS does not improve the results. The biggest advantage of ZigBee GBAS is its low cost and low power consumption. It is important in the task described in $[11,22,24]$. ZigBee operates on the $2.4 \mathrm{GHz}$ ISM (Industrial, Scientific and Medical) band which is available for all users for free. The communication capabilities of the ZigBee protocol allows GBAS to operate without prior knowledge of GBAS transmitter location (its position can be transmitted via the same device). This feature is a major improvement comparing to other GBAS systems [11]. The main disadvantage is the necessity to install additional infrastructure on the wharf and on the vessel. Multipath signal propagation problem may occur (which can be mitigated using antenna diversity) but this phenomena is not the subject of this paper [25]

The presented method can be applied for example in multiship trajectory planning $[7,9]$

Beside the PDOP factor, there are many other issues that must be taken into account in such system design [8].

\section{REFERENCES}

1. Szymkiewicz,R., "Finite-element method for the solution of the Saint Venant equations in an open channel network." Journal of hydrology 122.1 (1991): 275-287.

2. Tejchman, J., "Technical concept to prevent the silo honking." Powder Technology 106.1 (1999): 7-22.

3. Tsui, J., "Fundamentals of global positioning system receivers.” New York, NY, USA:: Wiley-Interscience, 2000.

4. Bielewicz, E., Górski, J.,"Shells with random geometric imperfections simulation-based approach." International journal of non-linear mechanics 37.4 (2002): 777-784.

5. Hegarty, C.J.,Chatre, E. "Evolution of the global navigation satellitesystem (gnss).” Proceedings of the IEEE 96.12 (2008): 1902-1917.

6. Kowalczyk, K., Rapiński, J.,Mroz, M., “Analysis of vertical movements modelling through various interpolation techniques.” Acta Geodyn. Geomater 7.4 (2010): 160. 
7. Szlapczynski, R., "Evolutionary sets of safe ship trajectories: a new approach to collision avoidance." Journal of Navigation 64.1 (2011): 169-181.

8. Rapiński, J., “Pseudolite location error”, Environmental Engineering (2011), vol 3, 1488-1450.

9. Szlapczynski R.,Szlapczynska,J.,"On evolutionary computing in multi-ship trajectory planning." Applied Intelligence 37.2 (2012): 155-174.

10. Janowski, A.,Rapinski,J., "M-split estimation in laser scanning data modeling." Journal of the Indian Society of Remote Sensing 41.1 (2013): 15-19.

11. Kaliński, K.J., Buchholz,C., "HILS for the design of threewheeled mobile platform motion surveillance system with a use of energy performance index." Solid State Phenomena. Vol. 198. Trans Tech Publications, 2013.

12. Rzepecka, Z. et al.,"Effect of additional distance measurements on satellite positioning." Acta Geodaetica et Geophysica 49.1 (2014): 35-44.

13. Leh,L., „ZigBee-based intelligent indoor positioning system soft computing." Soft Computing 18.3 (2014): 443-456.

14. Janowski, A., et al.,»Mobile indicators in GIS and GPS positioning accuracy in cities.» International Conference on Rough Sets and Intelligent Systems Paradigms. Springer International Publishing, 2014.

15. Janicka,J., Rapinski, J., "Filtering the results of ZigBee distance measurements with RANSAC algorithm", Acta Geodyn. Geomater., vol. 12, No. 3(179), 2015.

16. Wojcik, M.,Tejchman,J.. "Simulation of buckling process of cylindrical metal silos with flat sheets containing bulk solids." Thin-Walled Structures 93 (2015): 122-136.

17. Górski, J.,et al. „Effect of geometric imperfections on aluminium silo capacities.” Stahlbau 84.1 (2015): 52-57.

18. Rapiński,J.,"The application of ZigBee phase shift measurement in ranging", Acta Geodynamica et Geomaterialia, v. 12, No. 291780, 2015.

19. Rapinski, J.,Smieja M., "ZigBee Ranging using Phase Shift Measurements." Journal of Navigation 68.04 (2015): 665-677.

20. Nowak, A., „Dynamic GNSS Mission Planning Using DTM for Precise Navigation of Autonomous Vehicles." The Journal of Navigation (2016): 1-22.
21. Bharati,B., et al. "GPS C/A Code Multipath Error Estimation for Surveying Applications in Urban Canyon." Microelectronics, Electromagnetics and Telecommunications. Springer India, 2016. 135-142.

22. Kaliński,K. J.,Mazur,M. “Optimal control of 2-wheeled mobile robot at energy performance index." Mechanical Systems and Signal Processing 70 (2016): 373-386.

23. Gharghan, S.K., Rosdiadee,N.,Mahamod,I., "A Wireless Sensor Network with Soft Computing Localization Techniques for Track Cycling Applications." Sensors 16.8 (2016): 1043.

24. Kaliński,K.J., Mazur,M., "Optimal control at energy performance index of the mobile robots following dynamically created trajectories." Mechatronics (2016).

25. Shaoping,L., Xu.Ch., Zhong,R., "An Active RFID TagEnabled Locating Approach With Multipath Effect Elimination in AGV." IEEE Transactions on Automation Science and Engineering 13.3 (2016): 1333-1342.

26. Kaur, J.P.,Kaur,R., Mann,G.P., „Performance Analysis of ZigBee Mesh Networks Under Nodes Failure." Proceedings of the International Conference on Recent Cognizance in Wireless Communication \& Image Processing. Springer India, 2016.

27. Yunlong,T.,Wang,J., "Some remarks on PDOP and TDOP for multi-GNSS constellations." Journal of Navigation 69.01 (2016): 145-155.

28. Chrószcz, A., Łukasik,P.,Lupa, M. «Analysis of Performance and Optimization of Point Cloud Conversion in Spatial Databases.» IOP Conference Series: Earth and Environmental Science. Vol. 44. No. 5. IOP Publishing, 2016. 
CONTACT WITH THE AUTHOR

\author{
Artur Janowski \\ e-mail:artur.janowski@geodezja.pl \\ Institute of Geodesy, \\ Faculty of Geodesy, \\ Geospatial and Civil Engineering \\ University of Warmia and Mazury in Olsztyn \\ Poland; \\ Jacek Rapinski \\ e-mail: jacek.rapinski@uwm.edu.pl \\ Institute of Geodesy, \\ Faculty of Geodesy, \\ Geospatial and Civil Engineering \\ University of Warmia and Mazury in Olsztyn \\ Poland
}

